\title{
Improving End-of-Life Decision-Making About Resuscitation and Intubation
}

\author{
Sydney Morss Dy, MD, MSc and John F. P. Bridges, PhD \\ Department of Health Policy and Management, Johns Hopkins Bloomberg School of Public Health, Baltimore, MD, USA.
}

$\mathrm{J}$ Gen Intern Med 30(8):1049-50

DOI: $10.1007 / \mathrm{s} 11606-015-3287-5$

() Society of General Internal Medicine 2015

$\mathrm{D}$ ying patients receive numerous interventions, frequently with little likelihood of benefit. Overuse of invasive interventions has major quality of life, psychosocial and economic impact for patients, caregivers and health systems. Such treatment can be inconsistent with patient preferences and often occurs only because it is the default in the absence of active decision-making. Although documenting and following patients' end-of-life care preferences are recommended quality indicators $^{1}$ and patient safety strategies ${ }^{2}$ for patients with serious illness, discussions about these issues and documentation in the medical record usually do not occur.

When end-of-life discussions do occur, communication and decision-making are often suboptimal. Providers offer resuscitation without adequately explaining the procedure or its potential benefits and risks. A growing body of research, mostly in the setting of intensive care, demonstrates the effectiveness of communication and decision-making interventions, such as structured family meetings, for decreasing potentially inappropriate health care utilization at the end of life. ${ }^{3}$ However, these previous studies used provider-oriented interventions based on verbal communication. Decision support tools, including written, video and interactive options, are increasingly being developed and show promise for improving patients' knowledge, outcomes such as decision satisfaction, and the appropriateness of patient decisions.

In this issue of the Journal of General Internal Medicine, ElJawahri and colleagues ${ }^{4}$ report the results of a randomized trial of a simple and practical video education intervention to inform patients about resuscitation and intubation. This research team, part of the Video Images of Disease for Ethical Outcomes (VIDEO) consortium, used rigorous methods to develop and evaluate this decision support tool. In this study, they extend their previous research in the outpatient setting to the hospital in a randomized trial of 150 older patients with limited prognoses. The intervention included the video decision support tool and feedback about the patient's preferences to at least one physician on the patient's medical team. The hospital is a critical setting for such interventions since most

Published online April 18, 2015 patients do not have these discussions as outpatients and, even if they do occur, the decisions need to be updated with changes in clinical status.

Patients randomized to the intervention gained more knowledge about end-of-life decision options than controls, were twice as likely not to want resuscitation and had preferences for not having resuscitation and intubation statistically significantly more frequently documented and significantly more orders to withhold resuscitation (57 vs $19 \%$ ). In an unplanned subgroup analysis, at one-year follow up intervention patients received resuscitation and intubation less frequently than controls - among patients readmitted to the same hospital, only 2 (4\%) of the participants who stated they did not want intubation at baseline received intubation, compared to $7(22 \%)$ of controls.

Although few studies of decision support tools for patients with advanced illnesses have evaluated patient outcomes, a recent study of a similar video intervention in intensive care did not find any significant differences. ${ }^{5}$ In this study with a larger sample size (208 patients), the control group received a 16-page pamphlet and all participants participated in a code status discussion (compared to El-Jawahri and colleagues ${ }^{4}$ where the control group received no intervention at all). Median total knowledge scores were statistically significantly higher in the intervention group (13 compared to 10 on a 15-point scale), but there were no significant differences in documented preferences between the two groups $(5 \%$ in the intervention group compared to $6 \%$ in the control group changed preferences to do-not-resuscitate within $72 \mathrm{~h}$ ). This study may have found no impact because both groups received detailed information and a discussion or because of the study population (all already in intensive care at the time of intervention and not selected for patients with poor prognosis).

The study by El-Jawahri and colleagues ${ }^{4}$ has some limitations. The participants were mostly white; other patient groups should be studied since they may have different needs and preferences. Furthermore, most patients reported that they had not had any discussions about resuscitation with a physician since admission, so it is unclear what the impact of the video decision aid would have been compared to traditional provider-patient communication. Ideally, further research should evaluate processes by which increased documentation and preferences occur and how best to incorporate these types of interventions into usual clinical care. 
Communication about preferences, goals and care for serious illness extends beyond decisions about resuscitation and intubation $^{6}$ and should be a continuing conversation, addressing understanding of illness, goals, worries, and values. Communication should take place with family members as well as patients. ${ }^{7}$ Decisions about resuscitation should be made in the context of prognosis and values, and it is unknown how frequently this happened in this study. Research has also shown that patients frequently resist advance care planning, can find these discussions upsetting or harmful, and often do not understand the choices provided them, and providers often do not discuss or honor patients' wishes when they are documented. ${ }^{6,8}$

In addition, the presentation of resuscitation in the decision support tool may suggest that patient autonomy is paramount, whereas many experts recommend that physicians should make recommendations about the appropriateness of resuscitation, as is done with almost all other medical interventions, or not offer resuscitation as an option when the prognosis and preferences for comfort care are clear. Giving patients information without context or a recommendation gives the illusion that death is a choice. Providers potentially should not offer or recommend against resuscitation when it is unlikely to be beneficial and should focus on the desired outcomes and how best to achieve those goals, rather than on educating patients about the mechanics of the resuscitation process. ${ }^{8}$

More research on how to increase communication about end-of-life issues and improve the quality of that communication using methods that can be implemented in actual practice is greatly needed. The study by El-Jawahri and colleagues ${ }^{4}$ is an important step in that direction and adds to a growing body of research that more and improved communication about these issues is possible, can be practically implemented and can improve outcomes. Future developments in decision support tools, such as interactive methods that can be incorporated into the electronic medical record in the course of clinical practice, ${ }^{9}$ as well as through electronic patient portals and "living" documents that can evolve over time and go beyond preferences for resuscitation and intubation to address other elements of communication about serious illness, may help to advance care in these areas.

This study also highlights a broader issue - how should we be measuring patient-centered outcomes of end-of-life care? ${ }^{10}$ While palliative and end-of-life care has historically been at the forefront of patient- and family- centeredness, most studies still focus on provider- or system-oriented outcomes, such as the resuscitation rates reported by El-Jawarhi and colleagues. The broader movement to patient-centered outcomes research is changing medical research and much more is needed in palliative and end-of-life care as well. It remains to be seen which outcomes should be measured to assess palliative care interventions and who should be engaged in defining and measuring them. Simply measuring rates of resuscitation and intubation will not be enough to change practice: we need to demonstrate how more and better decision-making improves outcomes, such as reducing suffering and improving dignity and quality of life, that matter for patients and their caregivers.

Corresponding Author: Sydney Morss Dy, MD, MSc; Department of Health Policy and ManagementJohns Hopkins Bloomberg School of Public Health, Rm 609, 624 N Broadway, Baltimore, MD 21205, USA (e-mail: sdy@jhsph.edu).

\section{REFERENCES}

1. Dy SM, Kiley KB, Ast $\mathbf{K}$, et al. Measuring what matters: top-ranked quality indicators for hospice and palliative care from the American academy of hospice and palliative medicine and hospice and palliative nurses association. J Pain Symptom Manage. Epub, 2015.

2. Shekelle PG, Pronovost PJ, Wachter RM, et al. The top patient safety strategies that can be encouraged for adoption now. Ann Intern Med. 2013;158(5 Pt 2):365-368. doi:10.7326/0003-4819-158-5-201303051-00001.

3. Fawole OA, Dy SM, Wilson RF, et al. A systematic review of communication quality improvement interventions for patients with advanced and serious illness. J Gen Intern Med. 2013;28(4):570-577. doi:10.1007/ S11606-012-2204-4

4. El-Jawahri A, Mitchell SL, Paasche-Orlow MK, et al. A Randomized Controlled Trial Of A CPR And Intubation Video Decision Support Tool For Hospitalized Patients. J Gen Intern Med, 2015. [Spi 3200]

5. Wilson ME, Krupa A, Hinds RF, et al. A video to improve patient and surrogate understanding of cardiopulmonary resuscitation choices in the icu: a randomized controlled trial. Crit Care Med. 2015;43(3):621-629. doi: $10.1097 /$ Ccm.0000000000000749.

6. Billings JA. The need for safeguards in advance care planning. J Gen Intern Med. 2012;27(5):595-600.

7. Bernacki RE, Block SD. Communication about serious illness care goals: a review and synthesis of best practices. JAMA Internal Medicine. 2014; 174(12): 1994-2003.

8. Drought TS, Koenig BA. "Choice" in end-of-life decision making: researching fact or fiction? Gerontologist. 2002;42(Suppl 3):114-128.

9. Agoritsas T, Heen AF, Brandt $\mathbf{L}$, et al. Decision aids that really promote shared decision making: the pace quickens. BMJ. 2015;350:G7624.

10. Aslakson RA, Bridges JF. Assessing the impact of palliative care in the intensive care unit through the lens of patient-centered outcomes research. Curr Opin Crit Care. 2013;19(5):504-510. doi:10.1097/Mcc. Ob013e328364d50f. 